\title{
Pain management in total knee arthroplasty: efficacy of a multimodal opiate-free protocol
}

\author{
GIAN LUIGI CANATA, VALENTINA CASALE, ALFREDO CHIEY
}

Centre of Sports Traumatology, Koelliker Hospital, Turin, Italy

\begin{abstract}
Purpose: this study was conducted to identify the most effective method of postoperative pain management, comparing the intravenous opiate infusion protocol with the use of a single periarticular local anesthetic infiltration (LAI) in patients undergoing total knee arthroplasty (TKA) surgery.

Methods: 50 patients submitted to TKA surgery between 2013 and 2015 were divided into two groups. Buprenorphine was administered intravenously to the patients in Group A, while the Group B patients received a single periarticular LAI (ropivacaine and ketorolac) during surgery.

Pain was assessed using a visual analog scale (VAS) and the knee injury and osteoarthritis outcome score. Hemoglobin and hematocrit were measured in the early postoperative period and at 40 days post-surgery. Range of motion and inflammatory markers were also assessed. Statistical analysis was performed using Student's t-test.

Results: student's t-test showed no significant difference between the groups in functional outcomes or blood values, but a difference in VAS score on the day of surgery was found $(p<0.0001)$, in favor of Group B.

Conclusions: LAI considerably reduces postoperative pain, allowing rapid mobilization and accelerating functional recovery.

Level of evidence: Level I, prospective single-blind randomized trial.
\end{abstract}

\footnotetext{
Corresponding Author:

Gian Luigi Canata, MD

Centre of Sport Traumatology, Koelliker Hospital

Corso Galileo Ferraris 247, 10134 Turin, Italy

studio@ortosport.it
}

Keywords: arthroplasty, knee, local anesthetic infiltration, opiate, pain management.

\section{Introduction}

Total knee arthroplasty (TKA) is among the most successful orthopaedic procedures in treating knee osteoarthritis and improving quality of life (1).

However, arthroplasty procedures, especially knee replacements, are commonly associated with severe postoperative pain (2-4).

Pain after TKA may delay early mobilization, prolong hospitalization, and increase the risk of postoperative complications, such as knee stiffness, anterior knee pain and delayed recovery of strength $(1,5)$.

It is essential to achieve adequate postoperative pain control, starting from as early as the intraoperative phase (6). Increasing evidence shows that the anesthesia and analgesia agents administered in the perioperative period may affect the rates of surgical site infection, urinary retention, nausea and vomiting (7).

Essentially, anesthesia and pain management protocols consist of either general anesthesia combined with femoral nerve block (FNB), or spinal anesthesia associated with morphine (8); recently, patient-controlled analgesia has been widely reported, because of its therapeutic effects and safety (9).

In view of the huge variety of available procedures and agents, it is important, when seeking to obtain the ideal postoperative management protocol, to consider the possible collateral effects of each single treatment. The adverse effects that may occur, especially when different agents are combined, are nausea, emesis, excessive sedation, hypotension and inhibition of respiration (10).

Because of the wide range of pain relief methods that 
exist, the ideal multimodal analgesia may be achieved by combining different drugs using different administration routes in order to avoid side effects and complications and improve patient safety (9), as recommended by the American Society of Anesthesiologists practice guidelines for acute pain management (11). To ensure a proper approach to postoperative analgesia, it is crucial to consider the preoperative comorbidities commonly present in patients undergoing TKA. In particular, preoperative psychological comorbidities, such as anxiety, depression or negative mood, have been significantly associated with acute postoperative pain after TKA (12); furthermore, preoperative antidepressant use and opioid use have each been shown to be among the significant predictors of moderate to severe acute postoperative pain (13).

Several studies have suggested that the administration of selective COX-2 inhibitors may reduce postoperative pain and consumption of opioid analgesics after TKA, thus improving the active range of motion (ROM) in the immediate postoperative period (14). Over recent years, perioperative local anesthetic infiltration (LAI) has emerged as one of the preferred techniques for postoperative pain management. A systematic review by Marques et al. reported that lower pain levels at rest at 24 hours and during activity at 48 hours have been recorded in patients receiving LAI after TKA; indeed, these patients required less opioid medication, and showed a reduction of opioid-related collateral effects such as vomiting and nausea (15).

The aim of this study was to identify the most effective method of postoperative pain management comparing the intravenous opiate infusion protocol with a single periarticular LAI in patients submitted to TKA surgery.

\section{Methods}

\section{Participants}

This prospective single-blind randomized study was conducted in 50 patients (11 males and 39 females) with a mean age of 73 years (range, 55-84), submitted to TKA surgery between 2013 and 2015. Patients undergoing revision surgery were excluded from the study.

The patients were divided into two groups (A and B) each comprising 25 cases. Gender, age, body mass index (BMI), knee alignment and possible knee laxity were recorded preoperatively as baseline characteristics.

\section{Interventions}

All the surgical procedures were performed by the same surgeon at a single hospital center. All the patients underwent intraarticular injection of tranexamic acid (TXA) intraoperatively (Fig. 1); tourniquet was inflated in all cases to a pressure of $100 \mathrm{mmHg}$ above systolic arterial pressure.

In Group $\mathrm{A}$, an intravenous infusion of $0.3 \mathrm{mg}$ of buprenorphine in $250 \mathrm{cl}$ of normal saline, delivered at a rate of $20 \mathrm{ml} / \mathrm{h}$, was given within the first 12 hours postoperatively. In Group B, patients received a single periarticular LAI (2 vials of ropivacaine $10 \mathrm{mg} / \mathrm{dl}$ and 1 vial of ketorolac $30 \mathrm{mg} / \mathrm{dl}$ ) during surgery (Fig. 2). Subsequently, etoricoxib $90 \mathrm{mg}$ was administered orally, within the first 24 hours. Paracetamol $1 \mathrm{~g}$ was infused every 6 hours during the day after the surgery in both groups. No drainage procedures were performed after surgery. Manual lymphatic drainage was started during the first postoperative hours in both groups.

\section{Outcome measurements}

All patients were examined using the Knee Injury and Osteoarthritis Outcome Score (KOOS) at the baseline assessment and at clinical follow-up. A visual analog scale (VAS) was used to assess the pain levels on the day of surgery, within 1 week and 40 days after surgery. Hemoglobin and hematocrit were measured within 1 week following the surgery to evaluate blood loss. At 40 days after surgery, inflammatory markers, specifically erythrocyte sedimentation rate, and C-reactive protein, were assessed, as was the postoperative range

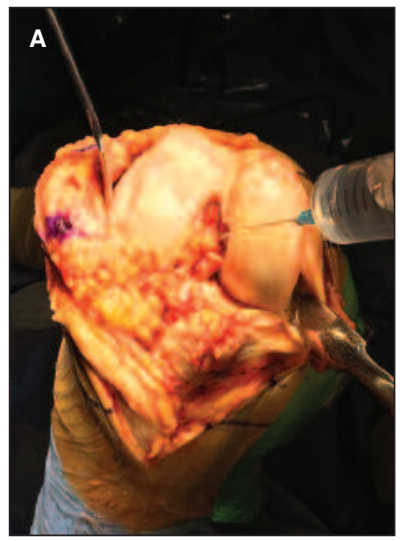

Figure 1. Topical application of tranexamic acid, before starting osteotomies (A) and before deflating the tourniquet and starting the wound closure (B). 
of motion (ROM) as a clinical measure.

\section{Data analysis}

Student's t-test was used to compare the results in the two groups. A p-value of less than 0.05 was considered statistically significant. All data were expressed as mean values with standard deviation (SD).

\section{Results}

Descriptive statistics of the two groups revealed no differences in age, gender, incidence of obesity, pre-

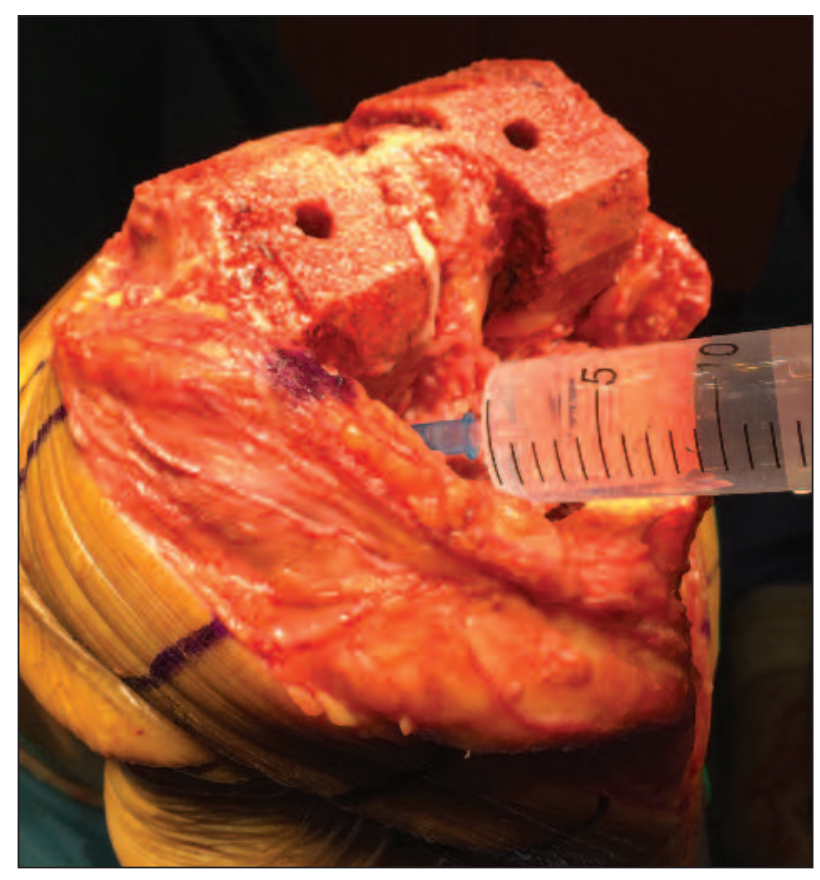

Figure 2. Local infiltration analgesia (LAI) of soft tissues with ropivacaine and ketorolac, before implanting the prosthesis. operative knee alignment or presence of knee medial ligament laxity (Tab. 1).

The mean follow-up was 4 months (range, 2-6 months). Student's t-test showed no significant difference in KOOS and ROM outcomes between the two groups.

Similarly, blood values and inflammatory markers did not differ significantly between the two groups, and neither did the pain assessment, with the exception of the VAS score on the day of surgery. This value was significantly lower in group B ( $\mathrm{p}$-value $=0.0001)$, a finding attributable to the action of periarticular LAI, which prolongs the analgesic effect in the immediate postoperative hours. Red blood cell transfusion was necessary in only one patient in each group.

\section{Discussion}

The combination of multimodal analgesia, preemptive analgesia and neuraxial anesthesia may be supplemented by peripheral nerve block (PNB), LAI or both.

Several studies have already tried to identify which of these two procedures is more effective. Kurosaka et al. observed a significant superiority of LAI in terms of VAS scores on the first day after surgery, but no differences in postoperative complications between LAI and FNB (4).

Wang et al. reviewed this issue, pointing out that LAI has rapidly gained popularity because of its simplicity and effectiveness, and identifying several studies that demonstrate comparable or even better results with LAI as opposed to FNB in postoperative management (16). In particular, although single-shot FNB provided adequate pain relief in the early postoperative period (at 12 hours), single-shot LAI provided better pain control than FNB at 24 hours, suggesting that the

Table 1. Patient demographics and baseline characteristics.

\begin{tabular}{lcc}
\hline & GROUP A $(\mathbf{n}=\mathbf{2 5})$ & GROUP B (n= 25) \\
Sex $(\mathrm{M} / \mathrm{F})$ & $4 / 21$ & $7 / 18$ \\
Side $(\mathrm{R} / \mathrm{L})$ & $14 / 11$ & $14 / 11$ \\
Mean age (years) & 74 (range $55-84)$ & 72 (range $64-84)$ \\
Patients with BMI $\geq 30$ & 19 & 17 \\
Knee alignment & 9 normal, 11 varus, 5 valgus & 9 normal, 8 varus, 8 valgus \\
Patients with medial knee laxity & 1 & 0 \\
\hline
\end{tabular}

$B M I=$ body mass index. 
Table 2. Baseline and follow-up outcomes.

GROUP A ( $\mathrm{n}=25)$

(Mean $\pm \mathrm{SD})$

$$
\begin{gathered}
10.9 \pm 1.1 \\
31.4 \pm 6.1 \\
40.4 \pm 19.1 \\
5.5 \pm 9.5 \\
\\
3.2 \pm 1.7 \\
1.2 \pm 1.6 \\
2.1 \pm 2.3 \\
0^{\circ}-0^{\circ}-110^{\circ} \\
\\
40.8 \pm 19.4 \\
75.6 \pm 19.5
\end{gathered}
$$

GROUP B ( $\mathrm{n}=25)$

(Mean \pm SD)

$$
\begin{gathered}
11.4 \pm 1.2 \\
32.8 \pm 2.9 \\
35.5 \pm 21.3 \\
4.3 \pm 4.6 \\
1.1 \pm 1.8 \\
0.7 \pm 1.3 \\
1 \pm 1.8 \\
0^{\circ}-0^{\circ}-110^{\circ} \\
\\
42.1 \pm 17.8 \\
79.7 \pm 19.7
\end{gathered}
$$

$H b=$ hemoglobin; $H t=$ hematocrit; $E S R=$ erythrocyte sedimentation rate; $C R P=\mathrm{C}$-reactive protein; VAS=Visual Analog Scale; $R O M=$ range of motion; $K O O S=$ Knee Injury and Osteoarthritis Outcome Score; $S D=$ standard deviation.

analgesic effect of single-shot LAI was longer lasting than that of FNB. Furthermore, PNB usually increases the frequency of falls, as a result of decreased quadriceps strength. Falls are dangerous when starting early mobilization after TKA, and may lengthen the hospital stay (17).

Yu et al. described the effects of intraoperative periarticular infiltration of liposomal bupivacaine compared to the FNB procedure (18). A liposomal cytarabine delivery agent was added to the anesthetic bupivacaine, extending its analgesic effect. The Authors reported a reduced use of narcotics in the LAI group, comparable pain between the two groups, and a quicker functional recovery in the LAI than in the FNB group.

There is still debate over the molecules that should be injected in a LAI. Epinephrine has emerged as one of the most frequently injected substances, mostly because of its potential to reduce toxic blood concentrations in comparison with other agents injected; it decreases the clearance and distribution processes into the blood flow of those substances and it also reduces the risk of bleeding (19). On the other hand, anesthetic drugs like ropivacaine have been shown to have vasoconstrictive properties (20), while epinephrine may cause local and systemic adverse effects, such as tissue necrosis and increased risk of infection (21). For this reason, the use of epinephrine has recently been losing consensus in favor of the isolated use of local anesthetics and non-steroidal anti-inflammatory drugs (NSAIDs). In a prospective randomized study, Villatte et al. showed that including epinephrine in the LAI does not significantly reduce peri- and postoperative bleeding (22).

Several studies have demonstrated that a single bolus of LAI may be more effective than continuous wound catheter infusion in controlling pain (23), and Villatte et al. have underlined that improvements in bleeding are possible, especially when ketorolac is added to the LAI solution (22). This NSAID, if added to the LAI solution, may result in a significant reduction of intravenous morphine consumption, and a decrease in pain intensity at rest and during activity, especially in the first 48 hours after surgery (24). However, it is well known that NSAIDs and cyclooxygenase inhibitors carry a higher risk of acute and chronic renal impairment (25). Older age in patients undergoing TKA, as well as treatments with diuretics, angiotensin-converting enzyme inhibitors or angiotensin II-receptor blocking agents are further risk factors for renal function impairment (26). Hence the importance of avoiding an excessive dose of NSAIDs, and choosing an appropriate mode of administration.

Affas et al. demonstrated that the maximal plasma concentration of ketorolac after a single LAI following total hip arthroplasty was comparable to that obtained after an intramuscular infiltration of the same drug at the same dose (27). 
This finding does not exclude the risk of NSAIDrelated renal adverse effects, even after a single LAI, but it helps to dispel questions over how safe the use of injectable NSAIDs is.

Bleeding control is a fundamental aspect of the perioperative clinical management. It has been reported that surgical procedures such as TKA may cause blood loss of more than one liter, resulting in longer hospital stays, delayed rehabilitation and poor subjective outcomes for the patient (28). If blood transfusions become necessary, this increases the costs of the treatment and the risk of periprosthetic infections (29).

As regards postoperative blood loss management, there are several possibilities, such as controlled hypotension and hemodilution, that can be considered in order to reduce transfusion requirements; recently, pharmacological agents like TXA have met with general consensus. TXA reduces blood loss through inhibition of clot degradation and fibrinolysis, which are usually increased after surgical procedures (30).

Topical applications of TXA have been shown to be very effective in controlling bleeding, especially when performed peri- and intraoperatively $(31,32)$. Finally, oral administration of TXA has been proved to be particularly useful before surgery $(33,34)$.

The peculiarity of the present study is that the administration of opioids has been totally omitted in favor of a combined analgesic procedure.

A further aspect to examine in relation to our multimodal management of postoperative pain is the role of manual lymphatic drainage. A limited ROM may frequently occur, secondary to edema, a clinical situation that sometimes evolves into arthrofibrosis (35). Trauma can result in reduction of tissue fluid movements and TKA can lead to muscle tightness; acute edema thus ensues, and the lymphatic system no longer drains properly. It has been demonstrated that early postoperative manual lymphatic drainage quickly restores interstitial fluid circulation, thus improving functional recovery and allowing rapid recovery of full active knee flexion; this concept is fundamental considering that poor knee flexion is likely to mean a longer rehabilitation period and a slower return to functional ROM (36).

The main limitations of the present study concern the relatively small number of patients and the fact the trial was performed at a single center. However, this single-blind trial reports data from patient-reported outcomes.

Although much remains to be done, we believe that this study may provide some fundamental insights concerning the use of multimodal analgesia for improving perioperative clinical practice.

In conclusion, the significant reduction of postoperative pain associated with the use of LAI allows rapid mobilization and accelerates functional recovery. Our multimodal protocol, consisting of spinal epidural anesthesia, a perioperative LAI and a perioperative infiltration of TXA, is an inexpensive and simple approach capable of giving excellent results; above all, it allowed us to completely abandon the use of opioid drugs. Nevertheless, high-quality multicenter randomized controlled trials are still needed, as well as studies with long-term follow-up.

\section{References}

1. Fang R, Liu Z, Alijiang A, et al. Efficacy of intra-articular local anesthetics in total knee arthroplasty. Orthopedics. 2015;38:e573-581.

2. Affas F, Nygårds EB, Stiller CO, et al. Pain control after total knee arthroplasty: a randomized trial comparing local infiltration anesthesia and continuous femoral block. Acta Orthop. 2011;82:441-447.

3. Essving P, Axelsson K, Kjellberg J, et al. Reduced morphine consumption and pain intensity with local infiltration analgesia (LIA) following total knee arthroplasty. Acta Orthop. 2010;81:354-360.

4. Kurosaka K, Tsukada S, Seino D, et al. Local infiltration analgesia versus continuous femoral nerve block in pain relief after total knee arthroplasty: a randomized controlled trial. J Arthroplasty. 2016;31:913-917.

5. Goyal N, McKenzie J, Sharkey PF, et al. The 2012 Chitranjan Ranawat award: intraarticular analgesia after TKA reduces pain: a randomized, double-blinded, placebo-controlled, prospective study. Clin Orthop Relat Res. 2013;471:64-75.

6. Niemeläinen M, Kalliovalkama J, Aho AJ, et al. Single periarticular local infiltration analgesia reduces opiate consumption until 48 hours after total knee arthroplasty. A randomized placebo-controlled trial involving 56 patients. Acta Orthop. 2014;85:614-619.

7. Moucha CS, Weiser MC, Levin EJ. Current strategies in anesthesia and analgesia for total knee arthroplasty. J Am Acad Orthop Surg. 2016;24:60-73.

8. Fischer HB, Simanski CJ, Sharp C, et al. A procedure-specific systematic review and consensus recommendations for postoperative analgesia following total knee arthroplasty. Anaesthesia. 2008;63:1105-1123.

9. Mammoto T, Fujie K, Mamizuka N, et al. Effects of postoperative administration of celecoxib on pain management in patients after total knee arthroplasty: study protocol for an open-label randomized controlled trial. Trials. 2016;17:45.

10. Mei S, Jin S, Chen Z, et al. Analgesia for total knee arthroplas- 
ty: a meta-analysis comparing local infiltration and femoral nerve block. Clinics (Sao Paulo). 2015;70:648-653.

11. American Society of Anesthesiologists Task Force on Acute Pain Management. Practice guidelines for acute pain management in the perioperative setting: an updated report by the American Society of Anesthesiologists Task Force on Acute Pain Management. Anesthesiology. 2012;116:248-273.

12. Roth ML, Tripp DA, Harrison MH, et al. Demographic and psychosocial predictors of acute perioperative pain for total knee arthroplasty. Pain Res Manag. 2007;12:185-194.

13. Liu SS, Buvanendran A, Rathmell JP, et al. Predictors for moderate to severe acute postoperative pain after total hip and knee replacement. Int Orthop. 2012;36:2261-2267.

14. Huang YM, Wang CM, Wang CT, et al. Perioperative celecoxib administration for pain management after total knee arthroplasty - a randomized, controlled study. BMC Musculoskelet Disord. 2008;9:77.

15. Marques EM, Jones HE, Elvers KT, et al. Local anaesthetic infiltration for peri-operative pain control in total hip and knee replacement: systematic review and meta-analyses of short- and long-term effectiveness. BMC Musculoskelet Disord. 2014;15:220.

16. Wang C, Cai XZ, Yan SG. Comparison of periarticular multimodal drug injection and femoral nerve block for postoperative pain management in total knee arthroplasty: a systematic review and meta-analysis. J Arthroplasty. 2015;30:1281-1286.

17. Johnson RL, Kopp SL, Hebl JR, et al. Falls and major orthopaedic surgery with peripheral nerve blockade: a systematic review and meta-analysis. Br J Anaesth. 2013;110: 518-528.

18. Yu S, Szulc A, Walton S, et al. Pain control and functional milestones in total knee arthroplasty: liposomal bupivacaine versus femoral nerve block. Clin Orthop Relat Res. 2016. doi: 10.1007/s11999-016-4740-4

19. Schotanus MG, Bemelmans YF, van der Kuy PH, et al. No advantage of adrenaline in the local infiltration analgesia mixture during total knee arthroplasty. Knee Surg Sports Traumatol Arthrosc. 2015. doi: 10.1007/s00167-015-3723-4.

20. Ratajczak-Enselme M, Estebe JP, Rose FX, et al. Effect of epinephrine on epidural, intrathecal, and plasma pharmacokinetics of ropivacaine and bupivacaine in sheep. $\mathrm{Br} J$ Anaesth. 2007;99:881-890.

21. Piper SL, Kramer JD, Kim HT, et al. Effects of local anesthetics on articular cartilage. Am J Sports Med. 2011;39:22452253.

22. Villatte G, Engels E, Erivan R, et al. Effect of local anaesthetic wound infiltration on acute pain and bleeding after primary total hip arthroplasty: the EDIPO randomised controlled study. Int Orthop. 2016;40:2255-2260.
23. Chen DW, Hsieh PH, Huang KC, et al. Continuous intraarticular infusion of bupivacaine for post-operative pain relief after total hip arthroplasty: a randomized, placebo-controlled, double-blind study. Eur J Pain. 2010;14:529-534.

24. Andersen KV, Nikolajsen L, Haraldsted V, et al. Local infiltration analgesia for total knee arthroplasty: should ketorolac be added? Br J Anaesth. 2013;111:242-248.

25. Clive DM, Stoff JS. Renal syndromes associated with nonsteroidal antiinflammatory drugs. N Engl J Med. 1984;310: 563-572.

26. Loboz KK, Shenfield GM. Drug combinations and impaired renal function - the 'triple whammy'. Br J Clin Pharmacol. 2005;59:239-243.

27. Affas F, Eksborg S, Wretenberg P, et al. Plasma concentration of ketorolac after local infiltration analgesia in hip arthroplasty. Acta Anaesthesiol Scand. 2014;58:1140-1145.

28. Sehat KR, Evans R, Newman JH. How much blood is really lost in total knee arthroplasty? Correct blood loss management should take hidden loss into account. Knee. 2000;7:151155.

29. Friedman R, Homering M, Holberg G, et al. Allogeneic blood transfusions and postoperative infections after total hip or knee arthroplasty. J Bone Joint Surg Am. 2014;96:272-278.

30. Melvin JS, Stryker LS, Sierra RJ. Tranexamic acid in hip and knee arthroplasty. J Am Acad Orthop Surg. 2015;23:732-740.

31. Panteli M, Papakostidis C, Dahabreh Z, et al. Topical tranexamic acid in total knee replacement: A systematic review and meta-analysis. Knee. 2013;20:300-309.

32. Chimento GF, Huff T, Ochsner JL Jr, et al. An evaluation of the use of topical tranexamic acid in total knee arthroplasty. J Arthroplasty. 2013;28(8 Suppl):74-77.

33. Alipour M, Tabari M, Keramati M, et al. Effectiveness of oral tranexamic acid administration on blood loss after knee arthroplasty: A randomized clinical trial. Transfus Apher Sci. 2013;49:574-577.

34. Zohar E, Ellis M, Ifrach N, et al. The postoperative bloodsparing efficacy of oral versus intravenous tranexamic acid after total knee replacement. Anesth Analg. 2004;99:16791683.

35. Ebert JR, Joss B, Jardine B, et al. Randomized trial investigating the efficacy of manual lymphatic drainage to improve early outcome after total knee arthroplasty. Arch Phys Med Rehabil. 2013;94:2103-2111.

36. Davies DM, Johnston DW, Beaupre LA, et al. Effect of adjunctive range-of-motion therapy after primary total knee arthroplasty on the use of health services after hospital discharge. Can J Surg. 2003;46:30-36. 